\title{
Multi-channel high-resolution spectrometer for analytical studies
}

\section{terahertz}

\author{
V. Vaks ${ }^{1,2}$, E. Domracheva ${ }^{1,2}$, S. Pripolzin ${ }^{1}$, M. Chernyaeva ${ }^{1,2, *}$, and A. Yablokov ${ }^{1,2}$ \\ ${ }^{1}$ Institute for Physics of Microstructures RAS, 603087 Nizhny Novgorod, Russia \\ ${ }^{2}$ Lobachevsky State University of Nizhni Novgorod, 603950 Nizhny Novgorod, Russia
}

\begin{abstract}
A method of multi-channel $\mathrm{THz}$ spectroscopy and a sample device that embodies the method were developed and described by the authors. The device has two independent $\mathrm{THz}$ radiation sources and a single receiving module. The novel method allows detecting preliminary and short-living chemical compounds and therefore to studychemical reaction dynamics. This has been shown by a series of test experiments. The method can be applied to researches in Physics, Chemistry, Astronomy, Medicine, and Biology.
\end{abstract}

The most important and fundamental studies in Chemistry, Biology, and Medicine are based on watching the ways chemical reactions follow. Studying of intermediate and shortliving chemical compounds can be conducted with the method of multi-channel terahertz (THz) spectroscopy. The $\mathrm{THz}$ spectrometer is based on the method of transient spectroscopy that utilizes the free polarization decay within a gaseous mixture. It can be described as follows. The gas sample polarizes, under the influence of the $\mathrm{THz}$ radiation at the gas resonant frequency. Once the radiation generator frequency is changed to a nonresonant one, the polarized gas emits the very same radiation as the one the polarization has been induced by. This is performed via generating of a specific probing radiation. Its frequency or phase is being switched periodically. If the radiation frequency is resonant, it induces and destroys a macroscopic polarization within the gas. The spectrometer that works this way has the sensitivity close to the theoretical limit $[1,2]$. Its resolution is bound by Doppler line broadening. The sensitivity remains high at a significant decrease of the sample gas pressure (up to hundredths or thousandths of a Torr); meanwhile low pressure provides high spectral resolution, as the collision broadening diminishes. The phaseswitching working mode allows conducting radiation absorption measurements at a steady frequency. That is needed to study how the selected substance concentration is changing in the measuring cavity [1].

To analyse the concentration dynamics of two substances simultaneously, a new spectrometer has been proposed. The device has two independent $\mathrm{THz}$ radiation sources and a single receiving module [3]. The two-channel spectrometer schematic is shown at Fig. 1. There are two independent radiation sources that emit at $115-178 \mathrm{GHz}$ and $330-390$ $\mathrm{GHz}$ frequency bands. The sources are based on backward wave oscillators (BWO). The moments of their radiation phase switching are separated in time, so that the resulting gas

*Corresponding author: stsav012@gmail.com 
polarization decays before the phase of another radiation source is switched. This results in receiving signals that are separated in time, too. As the phase switching happens quite frequently, the absorptions are measured almost simultaneously.

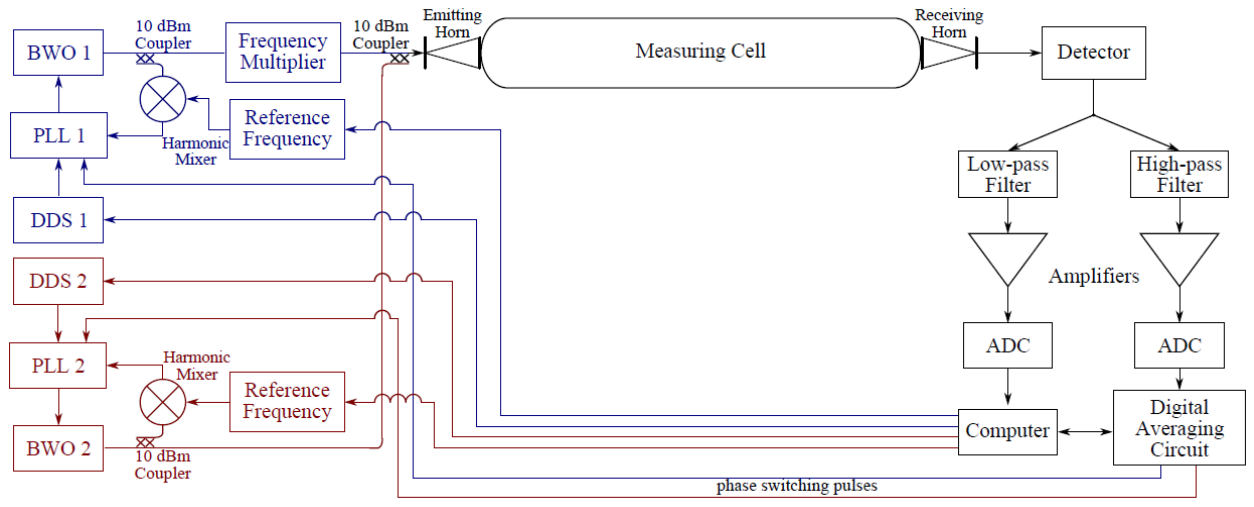

Fig. 1. The two-channel spectrometer schematic; here BWOs stand for backward wave oscillators, DDSs are direct digital synthesizers, PLLs are phase-locked loops.

We have performed test measurements using a mixture of ethanol and hydrogen peroxide. We observed a series of chemical reactions $\mathrm{C}_{2} \mathrm{H}_{5} \mathrm{OH}+\mathrm{H}_{2} \mathrm{O}_{2} \rightarrow \mathrm{CH}_{3} \mathrm{CHO}+$ $2 \mathrm{H}_{2} \mathrm{O}, \mathrm{CH}_{3} \mathrm{CHO}+\mathrm{H}_{2} \mathrm{O}_{2} \rightarrow \mathrm{CH}_{3} \mathrm{COOH}+\mathrm{H}_{2} \mathrm{O}$, and $2 \mathrm{CH}_{3} \mathrm{CHO}+\mathrm{O}_{2} \rightarrow 2 \mathrm{CH}_{3} \mathrm{COOH}$. They depended on a ratio of the initial compounds amounts. Besides, we have studied a mixture of formaldehyde and methanol that may be the products of some explosives slow decomposition. The measured absorption dynamics of the substances is shown at Fig. 2.

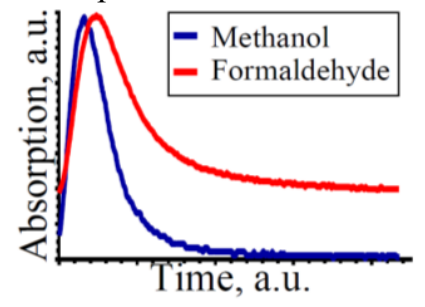

Fig. 2. Simultaneously measured absorption dynamics of methanol (bottom line) and formaldehyde (top line)
The experiments have proved that the method helps with real-time measurements of reagents concentrations. This can be applied to researches in Physics, Chemistry, Astronomy, Medicine, and Biology. The further development of the method will consist of using an IR radiation source together with $\mathrm{THz}$ ones. This should improve the reliability trustworthiness of the analysis and add non-polar molecules to the list of detectable compounds.

The article is the result of studies partly financed financially supported by the RFBR Grant no. 15-4202330 and by RSF (project no. 15-12-10035).

\section{References}

1. V.L. Vaks, A.B. Brailovsky, V.V. Khodos, Infrared \& Millimeter Waves 20, 883 (1999)

2. V.L. Vaks, Journal of Infrared, Millimeter and Terahertz Waves 33, 43 (2012)

3. V.L. Vaks, V.Y. Balakirev, M.B. Chernyaeva, E.G. Domracheva, S.I. Pripolzin, V.A. Anfertev, L.S. Revin, A.A. Yablokov, Proceedings of An International Joint Conference of The 9th Global Symposium on Millimeter-Waves (GSMM 2016) and The 7th ESAWorkshop on Millimetre-Wave Technology and Applications (2016) 